\title{
SIEVED ORTHOGONAL POLYNOMIALS. VII: GENERALIZED POLYNOMIAL MAPPINGS
}

\author{
JAIRO A. CHARRIS AND MOURAD E. H. ISMAIL
}

\begin{abstract}
Systems of symmetric orthogonal polynomials whose recurrence relations are given by compatible blocks of second-order difference equations are studied in detail. Applications are given to the theory of the recently discovered sieved orthogonal polynomials. The connection with polynomial mappings is examined. An example of a family of orthogonal polynomials having discrete masses in the interior of the spectrum is included.
\end{abstract}

\section{INTRODUCTION}

In this paper we study systems $\left\{p_{n}(x)\right\}$ of orthogonal polynomials whose recurrence relations are given by blocks of second-order difference equations

$$
\begin{aligned}
& x p_{n k}(x)=p_{n k+1}(x)+a_{n}^{(0)} p_{n k-1}(x) \\
& x p_{n k+1}(x)=p_{n k+2}(x)+a_{n}^{(1)} p_{n k}(x) \\
& \vdots \\
& x p_{n k+k-1}(x)=p_{n k+k}(x)+a_{n}^{(k-1)} p_{n k+k-2}(x), \quad n \geq 0, k \geq 2 .
\end{aligned}
$$

It is assumed that $k$ is a fixed integer, $k \geq 2$. We shall assume that the determinant

$$
\Delta_{n}(x ; 2, k-1)=\left|\begin{array}{cccccccc}
x & -1 & 0 & 0 & \ldots & 0 & 0 & 0 \\
-a_{n}^{(2)} & x & -1 & 0 & \cdots & 0 & 0 & 0 \\
0 & -a_{n}^{(3)} & x & -1 & \cdots & 0 & 0 & 0 \\
\vdots & \vdots & \vdots & \vdots & \vdots & \vdots & \vdots & \vdots \\
0 & 0 & 0 & 0 & \cdots & -a_{n}^{(k-2)} & x & -1 \\
0 & 0 & 0 & 0 & \ldots & 0 & -a_{n}^{(k-1)} & x
\end{array}\right|
$$

is independent of $n$ for $n \geq 0$. We assume

$$
a_{n}^{(j)}>0, \quad j=0,1, \ldots, k-1, n \geq 0,
$$

Received by the editors November 22, 1989 and, in revised form, December 27, 1989 and February 15, 1990.

1980 Mathematics Subject Classification (1985 Revision). Primary 42C05; Secondary 33A65.

Key words and phrases. Sieved polynomials, polynomial mappings, Julia sets, generalized polynomial mappings, $q$-ultraspherical polynomials, measures, moment problem, continued fractions.

Research partially supported by N.S.F. grants DMS8814026 and INT8803099. 
and also that, for some constant $M>0$,

$$
2 \sqrt{a_{n}^{(j)}} \leq M, \quad j=0,1, \ldots, k-1, n \geq 0 .
$$

The initial conditions

$$
p_{-1}(x)=0, \quad p_{0}(x)=1
$$

are assumed to hold throughout the paper. Observe that the polynomials generated by (1.1) and (1.5) are symmetric in the sense that $p_{n}(-x)=(-1)^{n} p_{n}(x)$.

Condition (1.4) insures (Chihara [11]) that the orthogonality measure $\mu$ of $\left\{p_{n}(x)\right\}$ (with $\mu(\mathbf{R})=1$ ) is unique and supp $\mu \subseteq[-M, M]$.

Special recurrence relations given by blocks of equations of the form (1.1) have appeared in the study of sieved symmetric polynomials (Al-Salam, Allaway, and Askey [1], Charris and Ismail [8], and Ismail [14, 15]), in the study of some processes of physics and chemistry (Wheeler [21], Slim [20], and Charris and Rodriguez [10]), and in the theory of orthogonal systems via polynomial mappings (Geronimo and Van Assche [12, 13]). Symmetric sieved polynomials of the first kind satisfy

$$
a_{n}^{(j)}=\frac{1}{4}, \quad j=2,3, \ldots, k-1, n \geq 0,
$$

so that only $a_{n}^{(0)}$ and $a_{n}^{(1)}$ may depend on $n$. In this case

$$
\Delta_{n}(x ; 2, k-1)=\frac{1}{2^{k-1}} U_{k-1}(x), \quad n \geq 0,
$$

where $U_{k-1}(x)$ is a Chebyshev polynomial of the second kind, and (1.2) holds. For symmetric sieved polynomials of the second kind we assume $k>2$,

$$
a_{n}^{(j)}=\frac{1}{4}, \quad j=1, \ldots, k-2, n \geq 0,
$$

and $\Delta_{n}(x ; 2, k-1)$ may depend on $n$. We will see, however, that the symmetric sieved polynomials of the second kind are numerators of approximants whose denominators are sieved polynomials of the first kind, and their properties can be deduced thereof.

The coefficients of the recursions of the sieved ultraspherical polynomials of [1] are

$$
a_{0}^{(0)}=a_{0}^{(1)}=\frac{1}{2} ; \quad a_{n}^{(0)}=\frac{n}{4(n+\lambda)}, \quad a_{n}^{(1)}=\frac{n+2 \lambda}{4(n+\lambda)}, \quad n \geq 1,
$$

for the polynomials of the first kind (with $a_{n}^{(j)}=\frac{1}{4}, j=2, \ldots, k-1, n \geq 0$ ) and

$$
a_{0}^{(0)}=\frac{1}{2}, \quad a_{0}^{(1)}=\frac{1}{4} ; \quad a_{n}^{(0)}=\frac{n}{4(n+\lambda)}, \quad a_{n-1}^{(k-1)}=\frac{n+2 \lambda}{4(n+\lambda)}, \quad n \geq 1,
$$

for those of the second kind (with $a_{n}^{(j)}=\frac{1}{4}, j=1,2, \ldots, k-2$ ). Those coefficients were obtained in [1] by a limiting process from the coefficients of the recurrence relation of the continuous $q$-ultraspherical polynomials of L. J. Rogers $[17,18,19]$. The current terminology of the continuous $q$-ultraspherical polynomials is due to Askey and Ismail [2, 3]. Askey and Ismail [2, 3] and Askey and Wilson [6] found the weight function of the continuous $q$-ultraspherical polynomials. A formal evaluation of the weight functions of the sieved ultraspherical 
polynomials of the first and second kinds is in [1]. The idea is to apply the same limiting processes to the weight function of the Rogers $q$-ultraspherical polynomials. The technical details of this approach in the case of the more general sieved Jacobi polynomials are in [5].

This work attempts to present a systematic study of symmetric polynomials defined by blocks of recurrence relations. We were motivated by the work on sieved orthogonal polynomials when (1.6) and (1.7) hold. In their very interesting work [13] Geronimo and Van Assche pointed out a connection between sieved orthogonal polynomials and the theory of polynomial mappings and polynomials orthogonal on Julia sets. They claimed, however, that all sieved orthogonal polynomials known at the time are covered by their approach using polynomial mappings. When we tried to verify the latter claim, we were pleasantly surprised to discover that the sieved Pollaczek polynomials [14] (see (1.11) below) did not fit the Geronimo-Van Assche theory. This led us to develop the different approach used in the present work.

The coefficients in the recurrences of the symmetric sieved Pollaczek polynomials of Ismail [14] are

$$
\begin{aligned}
& a_{0}^{(0)}=\frac{1}{2}, \quad a_{0}^{(1)}=\frac{\lambda}{2(\lambda+a)} ; \\
& a_{n}^{(0)}=\frac{n}{4(n+\lambda+a)}, \quad a_{n}^{(1)}=\frac{n+2 \lambda}{4(n+\lambda+a)}, \quad n \geq 1,
\end{aligned}
$$

for the polynomials of the first kind, and

$$
\begin{aligned}
& a_{0}^{(0)}=\frac{1}{2}, \quad a_{0}^{(1)}=\frac{1}{4} ; \\
& a_{n}^{(0)}=\frac{n}{4(n+\lambda+a)}, \quad a_{n-1}^{(k-1)}=\frac{n+2 \lambda}{4(n+\lambda+a)}, \quad n \geq 1, k>2,
\end{aligned}
$$

for those of the second kind. They were also obtained as limiting cases as $q \rightarrow e^{2 \pi i / k}$ from the coefficients of the recurrence relation of the $q$-Pollaczek polynomials of $[4,9]$. Generating functions for the sieved Pollaczek polynomials were obtained in [14] by letting $q \rightarrow e^{2 \pi i / k}$ in the generating functions of the $q$-Pollaczek polynomials. The values of the corresponding $J$-fraction were then determined by applying Darboux's method to the generating functions of the sieved Pollaczek polynomials and their numerators. Then Markov's Theorem and the Stieltjes-Perron inversion formula were used to determine the sought measures. This was also the approach followed by Charris and Ismail [9] in the nonsymmetric case. No attempt was made either in $[1,9,13]$ or [14] to establish the orthogonality measures directly from the recurrence relations.

The coefficients of the sieved ultraspherical polynomials are related to the coefficients

$$
A_{n}=\frac{(n+1)}{2(n+\lambda+1)} \text { and } B_{n}=\frac{(n+2 \lambda+1)}{2(n+\lambda+1)}
$$

of the recursion of the ultraspherical polynomials $\left\{C_{n}^{\lambda+1}(x)\right\}$ in the following manner:

$$
a_{0}^{(0)}=\frac{1}{2}, \quad a_{0}^{(1)}=\frac{1}{2} ; \quad a_{n}^{(0)}=\frac{1}{2} A_{n-1}, \quad a_{n}^{(1)}=\frac{1}{2} B_{n-1}, \quad n \geq 1,
$$

for the polynomials of the first kind, and

$$
a_{0}^{(0)}=\frac{1}{2}, \quad a_{0}^{(1)}=\frac{1}{4} ; \quad a_{n}^{(0)}=\frac{1}{2} A_{n-1}, \quad a_{n-1}^{(k-1)}=\frac{1}{2} B_{n-1}, \quad n \geq 1,
$$


for the polynomials of the second kind. In [8], Charris and Ismail extended this relationship to a general symmetric recurrence relation

$$
\begin{aligned}
& x R_{n}(x)=A_{n} R_{n+1}(x)+B_{n} R_{n-1}(x), \quad n \geq 0, \\
& R_{-1}(x)=0, \quad R_{0}(x)=1,
\end{aligned}
$$

where $A_{n}, B_{n}>0, n \geq 0$, to define sieved random walk polynomials of the first kind, $\left\{r_{n}(x)\right\}$, by (1.13), and sieved random walk polynomials of the second kind, $\left\{s_{n}(x)\right\}$, by (1.14). They also assumed that

$$
A_{n}+B_{n}=1, \quad n \geq 0,
$$

and established the explicit representations

$$
\frac{2^{n(k-1)} r_{n k}(x)}{A_{0} \cdots A_{n-1}}=R_{n}\left(T_{k}(x)\right)-R_{n-2}\left(T_{k}(x)\right), \quad n \geq 0,
$$

and

$$
\frac{2^{n(k-1)} s_{n k-1}(x)}{A_{0} \cdots A_{n-1}}=U_{k-1}(x) R_{n-1}\left(T_{k}(x)\right),
$$

where $T_{k}(x)$ and $U_{k-1}(x)$ are Chebyshev polynomials of the first and second kinds, respectively.

Formulae (1.17) and (1.18) led Geronimo and Van Assche in [13] to regard sieved symmetric polynomials as special cases of orthogonal systems obtained by means of changes of variables through polynomial mappings. Although sieved random walk polynomials are included in their model, many other important examples of sieved polynomials cannot be incorporated in their scheme of changing variables and polynomial mappings.

By $\left\{T_{n}(x)\right\}$ and $\left\{U_{n}(x)\right\}$ we denote, respectively, the systems of Chebyshev polynomials of the first and second kind, and

$$
\widehat{T}_{0}(x)=1, \quad \widehat{T}_{n+1}(x)=\frac{1}{2^{n}} T_{n+1}(x), \quad \widehat{U}_{n}(x)=\frac{1}{2^{n}} U_{n}(x), \quad n \geq 0,
$$

will be their corresponding systems of monic polynomials. The notation

$$
(a)_{n}= \begin{cases}1, & n=0, \\ a(a+1) \cdots(a+n-1), & n \geq 1,\end{cases}
$$

for shifted factorial will be used throughout.

This paper is arranged as follows. In $\S 2$ we formulate certain fundamental recurrence relations satisfied by the polynomials $\left\{p_{n}(x)\right\}$ in (1.1) and by the corresponding associated polynomials $\left\{p_{n}^{(i)}(x)\right\}$. The recurrence relations derived involve the determinants $\Delta_{n}(j, k-1)$ and $\Delta_{n}(2, j)$ (see (2.19)), and their usefulness will be illustrated in the subsequent sections. The idea of a generalized polynomial mapping is introduced in $\S 3$ (see (3.2)). The case of a polynomial mapping occurs if and only if the term in square brackets in (3.2) is the sum of the form $\delta_{n}+T(x)$, where $T(x)$ is a polynomial in $x$ independent of $n$ but $\left\{\delta_{n}\right\}$ is a sequence of constants. Our generalization allows the quantity in square brackets in (3.2) to be a polynomial in $x$ which may also depend on $n$ but is not necessarily of the form $\delta_{n}+T(x)$. In $\S 4$ the Stieltjes transform of the measure that $\left\{p_{n}(x)\right\}$ is orthogonal with respect to is identified in terms of asymptotic properties of certain polynomials. Similar results 
are also derived for the associated polynomials. In $\S 5$ we investigate the special cases of sieved orthogonal polynomials in some detail. As an application we give an explicit example of a system of orthogonal polynomials orthogonal on $[-1,1]$ with respect to a positive measure $d \mu$ such that the support of the absolutely continuous component is $[-1,1]$ but $d \mu$ has $k$ discrete masses in $(-1,1)$. We give explicit representations of the polynomials and also find the coefficients in the three term recurrence relation satisfied by the polynomials. Section 6 illustrates, in some detail, the differences between our approach and the polynomial mapping approach of Geronimo and Van Assche [13].

\section{BASIC RESULTS}

The system of equations (1.1) can be written in matrix form as

$$
\begin{aligned}
& {\left[\begin{array}{ccccccccc}
1 & 0 & 0 & 0 & \cdots & 0 & 0 & 0 & a_{n}^{(0)} \\
x & -1 & 0 & 0 & \cdots & 0 & 0 & 0 & 0 \\
-a_{n}^{(2)} & x & -1 & 0 & \cdots & 0 & 0 & 0 & 0 \\
0 & -a_{n}^{(3)} & x & -1 & \cdots & 0 & 0 & 0 & 0 \\
\cdot & \cdot & \cdot & \cdot & \cdots & \cdot & \cdot & \cdot & \cdot \\
0 & 0 & 0 & 0 & \cdots & -a_{n}^{(k-2)} & \cdot & \cdot & \cdot \\
0 & 0 & 0 & 0 & \cdots & -1 & 0 \\
0 & 0 & 0 & 0 & \cdots & 0 & -a_{n}^{(k-1)} & x & 0
\end{array}\right]\left[\begin{array}{c}
p_{n k+1} \\
p_{n k+2} \\
p_{n k+3} \\
p_{n k+4} \\
\cdot \\
\cdot \\
p_{n k+k-1} \\
p_{n k-1}
\end{array}\right]} \\
& =\left[\begin{array}{c}
x p_{n k} \\
a_{n}^{(1)} p_{n k} \\
0 \\
0 \\
0 \\
0 \\
p_{n k+k}
\end{array}\right] .
\end{aligned}
$$

We will write

$$
\Delta_{n}(i, j)=\Delta_{n}(x ; i, j):= \begin{cases}0, & j<i-2 \\ 1, & j=i-2 \\ x, & j=i-1\end{cases}
$$

and

$$
\begin{aligned}
\Delta_{n}(i, j) & =\Delta_{n}(x ; i, j) \\
& :=\left|\begin{array}{ccccccc}
x & -1 & 0 & 0 & \cdots & 0 & 0 \\
-a_{n}^{(i)} & x & -1 & 0 & \cdots & 0 & 0 \\
0 & -a_{n}^{(i+1)} & x & -1 & \cdots & 0 & 0 \\
\cdot & \cdot & \cdot & \cdot & \cdots & \cdot & \cdot \\
\cdot & \cdot & \cdot & \cdot & \cdots & \cdot & \cdot \\
0 & 0 & 0 & 0 & \cdots & -a_{n}^{(j)} & x
\end{array}\right|, \quad i \leq j .
\end{aligned}
$$

Solving (2.1) for $p_{n k+j}$ in terms of $p_{n k}$ and $p_{n k+k}$, by Cramer's rule, gives

$$
\Delta_{n}(2, k-1) p_{n k+j}=\Delta_{n}(2, j-1) p_{n k+k}+a_{n}^{(1)} a_{n}^{(2)} \cdots a_{n}^{(j)} \Delta_{n}(j+2, k-1) p_{n k}
$$

for $n \geq 0, j=0,1, \ldots, k-1$ (with $a_{n}^{(1)} \cdots a_{n}^{(j)}=1$ if $j=0$ ). Furthermore,

$$
a_{n}^{(0)} \Delta_{n}(2, k-1) p_{n k-1}=\left[x \Delta_{n}(2, k-1)-a_{n}^{(1)} \Delta_{n}(3, k-1)\right] p_{n k}-p_{n k+k} .
$$


Replacing $n$ by $n-1$ and $j$ by $k-1$ in (2.4) yields

$$
\begin{aligned}
& \Delta_{n-1}(2, k-1) p_{n k-1} \\
& \quad=\Delta_{n-1}(2, k-2) p_{n k}+a_{n-1}^{(1)} a_{n-1}^{(2)} \cdots a_{n-1}^{(k-1)} p_{n k-k}, \quad n \geq 1 .
\end{aligned}
$$

From (2.5) with $n=0$, it follows that

$$
p_{k}(x)=x \Delta_{0}(2, k-1)-a_{0}^{(1)} \Delta_{0}(3, k-1)=\Delta_{0}(1, k-1) .
$$

If $\left\{p_{n}(x)\right\}$ is a system of symmetric sieved polynomials of the first kind, so that $a_{n}^{(j)}=\frac{1}{4}, n \geq 0, j=2, \ldots, k-1$, then (2.4) will take the form

$$
U_{k-1}(x) p_{n k+j}(x)=2^{k-j} U_{j-1}(x) p_{n k+k}(x)+a_{n}^{(1)} 2^{2-j} U_{k-j-1}(x) p_{n k}(x),
$$

$j=1,2 \ldots, k-1$. Also

$$
p_{k}(x)=\frac{1}{2^{k-1}}\left[T_{k}(x)+\left(1-2 a_{0}^{(1)}\right) U_{k-2}(x)\right] .
$$

Now, for every $i, i=1,2, \ldots, k-1$, the system $\left\{p_{n}^{(i)}(x)\right\}$ of the associated polynomials of order $i$ satisfies

(2.10) $x p_{n k-i+j}^{(i)}=p_{n k-i+j+1}^{(i)}+a_{n}^{(j)} p_{n k-i+j-1}^{(i)}, \quad j=0,1, \ldots, k-1, n \geq 1$,

so that, solving for $p_{n k-i+j}^{(i)}$ in terms of $p_{n k-i}^{(i)}$ and $p_{n k+k-i}^{(i)}$, exactly as we did for (1.1), we obtain

$$
\begin{aligned}
& \Delta_{n}(2, k-1) p_{n k-i+j}^{(i)} \\
& \quad=\Delta_{n}(2, j-1) p_{n k+k-i}^{(i)}+a_{n}^{(1)} \cdots a_{n}^{(j)} \Delta_{n}(j+2, k-1) p_{n k-i}^{(i)}
\end{aligned}
$$

and

$$
\begin{aligned}
& a_{n}^{(0)} \Delta_{n}(2, k-1) p_{n k-i-1}^{(i)} \\
& \quad=\left[x \Delta_{n}(2, k-1)-a_{n}^{(1)} \Delta_{n}(3, k-1)\right] p_{n k-i}^{(i)}-p_{n k+k-i}^{(i)}
\end{aligned}
$$

for $j=0,1, \ldots, k-1, n \geq 1$.

On the other hand,

$$
\left[\begin{array}{ccccccc}
x & -1 & 0 & \ldots & 0 & 0 & 0 \\
-a_{0}^{(i+1)} & x & -1 & \cdots & 0 & 0 & 0 \\
\cdot & \cdot & \cdot & \cdots & \cdot & \cdot & \cdot \\
\cdot & \cdot & \cdot & \cdots & \dot{0} & \cdot & \cdot \\
0 & 0 & 0 & & -a_{0}^{(k-2)} & x & -1 \\
0 & 0 & 0 & \ldots & 0 & -a_{0}^{(k-1)} & x
\end{array}\right]\left[\begin{array}{c}
p_{0}^{(i)} \\
p_{1}^{(i)} \\
\cdot \\
\cdot \\
p_{k-i-2} \\
p_{k-i-}^{(i)}
\end{array}\right]=\left[\begin{array}{c}
0 \\
0 \\
\cdot \\
\cdot \\
0 \\
p_{k-1}^{(i)}
\end{array}\right]
$$

so that, by Cramer's rule,

$$
p_{j}^{(i)}(x)=\Delta_{0}(i+1, j+i-1), \quad j=0,1, \ldots, k-i .
$$

We observe that if $\left\{p_{n}(x)\right\}$ is a system of symmetric sieved polynomials of the first kind, then

$$
p_{j}^{(i)}(x)=2^{-j} U_{j}(x), \quad j=0,1, \ldots, k-i, i=1,2 \ldots, k-1,
$$


and (2.11) becomes

$$
\begin{array}{r}
U_{k-1}(x) p_{n k-i+j}^{(i)}=2^{k-j} U_{j-1}(x) p_{n k+k-i}^{(i)}+2^{2-j} a_{n}^{(1)} U_{k-j-1}(x) p_{n k-i}^{(i)}, \\
0<j<k .
\end{array}
$$

The associated polynomials of higher order are generated via

$$
x p_{n k+j}^{(l k+i)}=p_{n k+j+1}^{(l k k+i)}+a_{l+n}^{(j)} p_{n k+j-1}^{(l k+i)}, \quad l \geq 0,0 \leq i \leq k-1, n \geq 0,
$$

and the initial conditions

$$
p_{-1}^{(l k+i)}(x)=0, \quad p_{0}^{(l k+i)}(x)=1 .
$$

This yields

$$
p_{k}^{(l k)}=x \Delta_{l}(2, k-1)-a_{l}^{(1)} \Delta_{l}(3, k-1)=\Delta_{l}(1, k-1),
$$

which in the case of sieved polynomials of the first kind becomes

$$
p_{k}^{(l k)}(x)=2^{1-k}\left[T_{k}(x)+\left(1-2 a_{l}^{(1)}\right) U_{k-2}(x)\right] .
$$

Formulae (2.11), (2.12), and (2.14) also generalize to

$$
\begin{aligned}
& \Delta_{n+l}(2, k-1) p_{n k-i+j}^{(l k+i)} \\
& \quad=\Delta_{n+l}(2, j-1) p_{n k+k-i}^{(l k+i)}+a_{n+l}^{(1)} \cdots a_{n+l}^{(j)} \Delta_{n+l}(j+2, k-1) p_{n k-i}^{(l k+i)}, \\
& \begin{aligned}
a_{n+l}^{(0)} \Delta_{n+l}(2, k-1) p_{n k-i-1}^{(l k+i)} \\
\quad=\left[x \Delta_{n+l}(2, k-1)-a_{n+l}^{(1)} \Delta_{n+l}(3, k-1)\right] p_{n k-i}^{(l k+i)}-p_{n k+k-i}^{(l k+i)},
\end{aligned}
\end{aligned}
$$

which hold for $n \geq 1$, and

$$
p_{j}^{(l k+i)}(x)=\Delta_{l}(i+1, j+i-1),
$$

which holds for $0<i<k, j=0,1, \ldots, k-i$. In the case of sieved polynomials we have

$$
p_{j}^{(l k+i)}(x)=2^{-j} U_{j}(x), \quad j=0,1, \ldots, k-i, i=1,2, \ldots, k-1,
$$

and

$$
\begin{aligned}
U_{k-1}(x) p_{n k-i+j}^{(l k+i)}=2^{k-j} U_{j-1}(x) p_{n k+k-i}^{(l k+i)}+2^{2-j} a_{n+l}^{(1)} U_{k-j-1}(x) p_{n k-i}^{(l k+i)} & \\
& i, j=1,2, \ldots, k-1, n \geq 1 .
\end{aligned}
$$

\section{THE FUNDAMENTAL RECURRENCE RELATION AND THE LINK POLYNOMIALS}

Let $\left\{p_{n}(x)\right\}$ be given by (1.1) and the initial conditions (1.5). Let $\left\{P_{n}(x)\right\}$ be the polynomials given initially by

$$
P_{-1}(x)=0, \quad P_{0}(x)=1
$$

and generated by

$$
\begin{gathered}
{\left[x \Delta_{n}(2, k-1)-a_{n}^{(1)} \Delta_{n}(3, k-1)-a_{n}^{(0)} \Delta_{n-1}(2, k-2)\right] P_{n}(x)} \\
=P_{n+1}(x)+a_{n}^{(0)} a_{n-1}^{(1)} \cdots a_{n-1}^{(k-1)} P_{n-1}(x), \quad n \geq 0,
\end{gathered}
$$


where we define

$$
\Delta_{-1}(2, k-2):=0 \text {. }
$$

For each $n \geq 0, P_{n}(x)$ is a polynomial of degree $n k$. The polynomials $\left\{P_{n}(x)\right\}$ will be called the link polynomials of the blocks (1.1), $n \geq 0$. It follows from (2.5) and (2.6) that if (1.2) holds then $\left\{p_{n k}(x)\right\}$ is a solution of (3.2), and (1.5) insures that in such a case, and only then,

$$
p_{n k}(x)=P_{n}(x), \quad n \geq 0 .
$$

Since $p_{n k}(x)$ and $P_{n}(x)$ are identical under assumption (1.5), denoting $p_{n k}(x)$ by $P_{n}(x)$ will be useful in what follows.

For each $l \geq 0$, we define $\left\{P_{n}^{(l)}(x)\right\}$ recursively by

$$
P_{-1}^{(l)}(x)=0, \quad P_{0}^{(l)}(x)=1,
$$

and

$$
\begin{gathered}
{\left[x \Delta_{n+l}(2, k-1)-a_{n+l}^{(1)} \Delta_{n+l}(3, k-1)-a_{n+l}^{(0)} \Delta_{n+l-1}(2, k-2)\right] P_{n}^{(l)}(x)} \\
\quad=P_{n+1}^{(l)}(x)+a_{n+l}^{(0)} a_{n+l-1}^{(1)} \cdots a_{n+l-1}^{(k-1)} P_{n-1}^{(l)}(x), \quad n \geq 0 .
\end{gathered}
$$

Clearly, $P_{n}^{(0)}(x)=P_{n}(x)$. Furthermore, $\left\{P_{n}^{(l)}(x) ; n \geq 0\right\}$ and $\left\{P_{n-1}^{(l+1)}(x) ; n \geq\right.$ $0\}$ are linearly independent solutions of (3.6). In fact, their Casorati determinant is

$$
W\left(P_{n}^{(l)}(x), P_{n-1}^{(l+1)}(x)\right)=\left|\begin{array}{cc}
P_{n}^{(l)}(x), & P_{n-1}^{(l+1)}(x) \\
P_{n+1}^{(l)}(x), & P_{n}^{(l+1)}(x)
\end{array}\right|=\alpha_{n}^{(l),} \quad n \geq 0,
$$

where

$$
\alpha_{0}^{(l)}=1 ; \quad \alpha_{n}^{(l)}=\prod_{j=1}^{n} a_{j+l}^{(0)} \cdot a_{j+l-1}^{(1)} \cdots a_{j+l-1}^{(k-1)} \neq 0, \quad n>0 .
$$

We observe that for $i=1,2, \ldots, k-1$, the system $\left\{p_{n k+k-i}^{(i)}: n>0\right\}$ satisfies (3.6) with $l=1$. Hence

$$
p_{n k+k-i}^{(i)}(x)=A_{i} P_{n}^{(1)}(x)+B_{i} P_{n-1}^{(2)}(x), \quad n \geq 0 .
$$

Clearly, $A_{i}=p_{k-i}^{(i)}(x)$. On the other hand, from (3.6), with $l=1, n=0$, and (2.12) we get

$$
\begin{aligned}
p_{2 k-i}^{(i)}(x)= & p_{k-i}^{(i)}(x) P_{1}^{(1)}(x)+B_{i} \\
= & p_{k-1}^{(i)}(x)\left[x \Delta_{1}(2, k-1)-a_{1}^{(1)} \Delta_{1}(3, k-1)\right] \\
& \quad-a_{1}^{(0)} \Delta_{0}(2, k-2) p_{k-i}^{(i)}(x)+B_{i} \\
= & p_{2 k-i}^{(i)}(x)+a_{1}^{(0)}\left[\Delta_{1}(2, k-1) p_{k-i-1}^{(i)}(x)-\Delta_{0}(2, k-2) p_{k-i}^{(i)}(x)\right]+B_{i} .
\end{aligned}
$$

Hence

$$
\begin{aligned}
B_{i} & =a_{1}^{(0)}\left[\Delta_{0}(2, k-2) p_{k-i}^{(i)}(x)-\Delta_{0}(2, k-1) p_{k-i-1}^{(i)}(x)\right] \\
& =a_{1}^{(0)} W\left(p_{k-2}^{(1)}(x), p_{k-i-1}^{(i)}(x)\right)=a_{1}^{(0)} a_{0}^{(i)} \cdots a_{0}^{(k-1)} p_{i-2}^{(1)}(x),
\end{aligned}
$$


or, equivalently,

$$
B_{i}=a_{1}^{(0)} a_{0}^{(i)} \cdots a_{0}^{(k-1)} \Delta_{0}(2, i-2) .
$$

Therefore, for $i=1,2, \ldots, k-1, n \geq 0$,

$$
\begin{aligned}
p_{n k+k-i}^{(i)}(x)= & \Delta_{0}(i+1, k-1) P_{n}^{(1)}(x) \\
& +a_{1}^{(0)} a_{0}^{(i)} \cdots a_{0}^{(k-1)} \Delta_{0}(2, i-2) P_{n-1}^{(2)}(x) .
\end{aligned}
$$

In case $i=1,(3.9)$ reduces to

$$
p_{n k+k-1}^{(1)}(x)=\Delta_{0}(3, k-1) P_{n}^{(1)}(x)
$$

and when $i=2$ it reduces to

$$
p_{n k+k-2}^{(2)}(x)=\Delta_{0}(3, k-1) P_{n}^{(1)}(x)+a_{1}^{(0)} a_{0}^{(2)} \cdots a_{0}^{(k-1)} P_{n-1}^{(2)}(x) .
$$

Formula (3.9) can be generalized to

$$
\begin{aligned}
p_{n k+k-i}^{(l k+i)}(x)= & \Delta_{l}(i+1, k-1) P_{n}^{(l+1)}(x) \\
& +a_{l+1}^{(0)} a_{l}^{(i)} \cdots a_{l}^{(k-1)} \Delta_{l}(2, i-2) P_{n-1}^{(l+2)}(x),
\end{aligned}
$$

where $l \geq 0, i=1,2, \ldots, k-1$, and $n \geq 0$.

For symmetric sieved polynomials of the first kind (3.12) gives

$$
p_{n k+k-1}^{(l k+1)}(x)=2^{1-k} U_{k-1}(x) P_{n}^{(l+1)}(x)
$$

and

$$
\begin{array}{r}
p_{n k+k-i}^{(l k+i)}(x)=2^{i-k}\left[U_{k-i}(x) P_{n}^{(l+1)}(x)+2^{2-k} a_{l+1}^{(0)} U_{i-2}(x) P_{n-1}^{(l+2)}(x)\right], \\
i \geq 2 .
\end{array}
$$

We finally observe that the same line of argument yields

$$
\begin{aligned}
p_{n k+k}(x) & =\Delta_{0}(1, k-1) P_{n}^{(1)}(x)+a_{1}^{(0)} W\left(p_{k-2}^{(1)}, p_{k-1}\right) \\
& =\Delta_{0}(1, k-1) P_{n}^{(1)}(x)-a_{1}^{(0)} a_{0}^{(1)} \cdots a_{0}^{(k-1)} P_{n-1}^{(2)}(x), \quad n \geq 0 .
\end{aligned}
$$

\section{The Stieltjes transforms}

In this section we describe the Stieltjes transforms $X(x)$ of the distribution function of the orthogonality measure $d \mu$ of the polynomials $\left\{p_{n}(x)\right\}$. In some cases it is possible to recover $\mu$ from $X(x)$ via the Stieltjes-Perron inversion formula

$$
\int_{-\infty}^{\infty} f d \mu=\lim _{\varepsilon \rightarrow 0^{+}} \frac{1}{2 \pi i} \int_{-\infty}^{\infty}\{X(x-i \varepsilon)-X(x+i \varepsilon)\} f(x) d x,
$$

which holds for any continuous function $f$ on $\mathbf{R}$. The distribution function $\sigma$ of $\mu$,

$$
\sigma(x)=\int_{-\infty}^{x} d \mu=\mu((-\infty, x])
$$

is also given by

$$
\sigma(x)=\lim _{\varepsilon \rightarrow 0^{+}} \frac{1}{2 \pi i} \int_{-\infty}^{x}\{X(t-i \varepsilon)-X(t+i \varepsilon)\} d t
$$


We will establish some representations of $X(x)$ in terms of the link polynomials. These representations will be useful as we will see later in $\S 5$.

For the polynomials $\left\{p_{n}(x)\right\}$ as given by (1.1) and (1.5) and for their link polynomials $\left\{P_{n}(x)\right\}$ we have, from (3.4) and (3.10), that

$$
\frac{p_{n k-1}^{(1)}(x)}{p_{n k}(x)}=\Delta_{0}(2, k-1) \frac{P_{n-1}^{(1)}(x)}{P_{n}(x)} .
$$

Hence, $\lim _{n \rightarrow \infty}\left[P_{n-1}^{(1)}(x) / P_{n}(x)\right]$ exists for $x \notin[-M, M]$, and we have

$$
X(x)=\Delta_{0}(2, k-1) \lim _{n \rightarrow \infty} \frac{P_{n-1}^{(1)}(x)}{P_{n}(x)}, \quad x \notin[-M, M] .
$$

For symmetric sieved polynomials of the first kind (4.5) becomes

$$
X(x)=\frac{1}{2^{k-1}} U_{k-1}(x) \lim _{n \rightarrow \infty} \frac{P_{n-1}^{(1)}(x)}{P_{n}(x)} .
$$

Now we determine the Stieltjes transform of the associated polynomials $\left\{p_{n}^{(i)}(x)\right\}$. We examine first the case $i=1$. From (3.10) and (3.11) we obtain the following representation for $X(x)$,

$$
X(x)=\frac{1}{\Delta_{0}(2, k-1)}\left\{\Delta_{0}(3, k-1)+a_{1}^{(0)} a_{0}^{(2)} \cdots a_{0}^{(k-1)} \lim _{n \rightarrow \infty} \frac{P_{n-1}^{(2)}(x)}{P_{n}^{(1)}(x)}\right\}
$$

$x \notin[-M, M]$, which for sieved polynomials of the first kind reduces to

$$
X(x)=\frac{2}{U_{k-1}(x)}\left\{U_{k-2}(x)+\frac{a_{1}^{(0)}}{2^{k-2}} \lim _{n \rightarrow \infty} \frac{P_{n-1}^{(2)}(x)}{P_{n}^{(1)}(x)}\right\} .
$$

Formula (4.8) can be used to determine the Stieltjes transform of the measure of orthogonality of sieved polynomials $\left\{p_{n}(x)\right\}$ of the second kind. To see this define the system $\left\{q_{n}(x)\right\}$ of sieved polynomials of the first kind by

$$
\begin{aligned}
& q_{-1}(x)=0, \quad q_{0}(x)=1, \\
& x q_{k n+j}(x)=q_{k n+j+1}(x)+b_{n}^{(j)} q_{k n+j-1}(x), \quad n \geq 0,0 \leq j<k,
\end{aligned}
$$

where

$$
b_{0}^{(0)}=b_{0}^{(1)}=\frac{1}{2} ; \quad b_{n}^{(0)}=a_{n-1}^{(k-1)}, \quad b_{n}^{(1)}=a_{n}^{(0)}, \quad n \geq 1,
$$

and

$$
b_{n}^{(j)}=\frac{1}{4}, \quad j=2,3, \ldots, k-1, n \geq 0, k>2 .
$$

Then $p_{n}(x)=q_{n}^{(1)}(x), n \geq 0$. If $\left\{P_{n}(x)\right\}$ is the system of link polynomials of $\left\{q_{n}(x)\right\}$, then the Stieltjes transform of the measure that $\left\{p_{n}(x)\right\}$ is orthogonal with respect to, will be given by

$$
X(x)=\frac{2}{U_{k-1}(x)}\left\{U_{k-2}(x)+\frac{a_{0}^{(k-1)}}{2^{k-2}} \lim _{n \rightarrow \infty} \frac{P_{n-1}^{(2)}(x)}{P_{n}^{(1)}(x)}\right\} .
$$

Now we give an explicit formula for the Stieltjes transform of the measure of orthogonality of the associated polynomials of higher order of any system 
$\left\{p_{n}(x)\right\}$ satisfying (1.1). This will extend (4.7) to associated polynomials of higher order. Let $l \geq 0, i=0,1, \ldots, k-1$, and write

$$
F^{(l)}(x)=\lim _{n \rightarrow \infty} \frac{P_{n-1}^{(l+1)}(x)}{P_{n}^{(l)}(x)}
$$

where $\left\{P_{n}(x)\right\}$ is the system of link polynomials of $\left\{p_{n}(x)\right\}$. Then, if $X(x)$ denotes the Stieltjes transform of the orthogonality measure of $\left\{p_{n}^{(l k+i)}(x)\right\}$, we will have

$$
X(x)=\frac{\Delta_{l}(2, k-1) F^{(l)}(x)}{1+a_{l}^{(0)} \Delta_{l-1}(2, k-2) F^{(l)}(x)}, \quad l \geq 0, \quad i=0
$$

(4.15) $X(x)=\frac{\Delta_{l}(i+2, k-1)+a_{l+1}^{(0)} a_{l}^{(i+1)} \cdots a_{l}^{(k-1)} \Delta_{l}(2, i-1) F^{(l+1)}(x)}{\Delta_{l}(i+1, k-1)+a_{l+1}^{(0)} a_{l}^{(i)} \cdots a_{l}^{(k-1)} \Delta_{l}(2, i-2) F^{(l+1)}(x)}$,

$l \geq 0,0<i \leq k-2$, and

$$
X(x)=\frac{1+a_{l+1}^{(0)} \Delta_{l}(2, k-2) F^{(l+1)}(x)}{x+a_{l+1}^{(0)} a_{l}^{(k-1)} \Delta_{l}(2, k-3) F^{(l+1)}(x)}, \quad l \geq 0, \quad i=k-1 .
$$

For sieved polynomials of the first kind we obtain

$$
\begin{array}{r}
X(x)=\frac{1}{2^{k-1}} U_{k-1}(x) F^{(0)}(x), \quad X(x)=\frac{2^{1-k} U_{k-1}(x) F^{(l)}(x)}{1+2^{2-k} U_{k-2}(x) F^{(l)}(x)}, \\
l \geq 1, i=0 .
\end{array}
$$

$$
X(x)=\frac{2}{U_{k-1}(x)}\left\{U_{k-2}(x)+\frac{a_{l+1}^{(0)}}{2^{k-2}} F^{(l+1)}(x)\right\}, \quad \text { if } i=1,
$$

$$
X(x)=2 \frac{2^{k-2} U_{k-i-1}(x)+a_{l+1}^{(0)} U_{i-1}(x) F^{(l+1)}(x)}{2^{k-2} U_{k-i}(x)+a_{l+1}^{(0)} U_{i-2}^{\prime}(x) F^{(l+1)}(x)}, \quad \text { if } 1<i<k-1
$$

$$
X(x)=2 \frac{2^{k-2}+a_{l+1}^{(0)} U_{k-2}(x) F^{(l+1)}(x)}{2^{k-1} x+a_{l+1}^{(0)} U_{k-3}(x) F^{(l+1)}(x)}, \quad \text { if } i=k-1
$$

\section{SiEVED SYMMETRIC POLYNOMIALS}

Let $\left\{R_{n}(x)\right\}$ be the system of orthogonal polynomials given by (1.15) with $A_{n}, B_{n}>0, n \geq 0$. Define the coefficients in the recursion relation of the system $\left\{r_{n}(x)\right\}$ of sieved polynomials of the first kind of $\left\{R_{n}(x)\right\}$ by (1.13). The polynomials $\left\{r_{n}(x)\right\}$ are symmetric sieved polynomials and

$$
\begin{aligned}
& \Delta_{n}(2, k-1)=2^{1-k} U_{k-1}(x), \\
& \Delta_{n}(2, k-2)=\Delta_{n}(3, k-1)=2^{2-k} U_{k-2}(x) .
\end{aligned}
$$

Also,

$$
x \Delta_{0}(2, k-1)-a_{0}^{(1)} \Delta_{0}(3, k-1)=2^{1-k} T_{k}(x) .
$$


With

$$
P_{n}(x)=r_{n k}(x), \quad A_{-1}=0, \quad B_{-1}=1,
$$

(3.2) becomes

$$
\begin{aligned}
2^{1-k} & {\left[T_{k}(x)-\left(A_{n-1}+B_{n-1}-1\right) U_{k-2}(x)\right] P_{n}(x) } \\
& =P_{n+1}(x)+4^{1-k} A_{n-1} B_{n-2} P_{n-1}(x), \quad n \geq 0 .
\end{aligned}
$$

If

$$
Q_{n}(x)=\frac{2^{n k-n} P_{n}(x)}{A_{0} \cdots A_{n-1}}, \quad n>0, \quad Q_{0}(x)=1,
$$

then (5.4) becomes

(5.6) $\left[T_{k}(x)-\left(A_{n-1}+B_{n-1}-1\right) U_{k-2}(x)\right] Q_{n}(x)=A_{n} Q_{n+1}(x)+B_{n-2} Q_{n-1}(x)$.

Let $R_{n}^{\prime}(x), n \geq 0$, be the system of polynomials given by

$$
\begin{aligned}
& x R_{n}^{\prime}(x)=A_{n} R_{n+1}^{\prime}(x)+B_{n-2} R_{n-1}^{\prime}(x), \quad n \geq 0, \\
& R_{-1}^{\prime}(x)=0, \quad R_{0}^{\prime}(x)=1 .
\end{aligned}
$$

If the polynomials $\left\{R_{n}(x)\right\}$ are random walk polynomials, and only in that case, (5.6) reduces to

$$
T_{k}(x) Q_{n}(x)=A_{n} Q_{n+1}(x)+B_{n-2} Q_{n-1}(x), \quad n \geq 0 .
$$

Furthermore, for random walk polynomials we have

$$
R_{n}^{\prime}(x)=R_{n}(x)-R_{n-2}(x), \quad n \geq 1 .
$$

Hence, the following holds if and only if the polynomials $R_{n}(x)$ are random walk polynomials:

$$
\frac{2^{n k-n} r_{n k}(x)}{A_{0} \cdots A_{n-1}}=R^{\prime}\left(T_{k}(x)\right)=R_{n}\left(T_{k}(x)\right)-R_{n-2}\left(T_{k}(x)\right), \quad n \geq 1 .
$$

It is readily seen that $(5.10)$ is equivalent to

$$
P_{n}(x)=2^{1-k} \widehat{R}_{n}^{\prime}\left(T_{k}(x)\right),
$$

where $\widehat{R}_{n}^{\prime}(x), n \geq 0$, is the system of monic polynomials of $\left\{R_{n}^{\prime}(x)\right\}$. Formula (5.10) is (3.4) of [8].

On the other hand, from (3.13) we get

$$
r_{n k-1}^{(1)}(x)=2^{1-k} U_{k-1}(x) P_{n-1}^{(1)}(x),
$$

and the polynomials $\left\{P_{n}^{(1)}(x): n \geq 1\right\}$ satisfy

$$
\begin{aligned}
2^{1-k} & {\left[T_{k}(x)-\left(A_{n}+B_{n}-1\right) U_{k-2}(x)\right] P_{n}^{(1)}(x) } \\
& =P_{n+1}^{(1)}(x)+4^{1-k} A_{n} B_{n-1} P_{n-1}^{(1)}(x), \quad n \geq 0,
\end{aligned}
$$

so that if

$$
Q_{n}^{\prime}(x)=\frac{2^{n k-n} p^{(1)}(x)}{B_{0} \cdots B_{n-1}}
$$

then

$$
\left[T_{k}(x)-\left(A_{n}+B_{n}-1\right) U_{k-2}(x)\right] Q_{n}^{\prime}(x)=B_{n} Q_{n+1}^{\prime}(x)+A_{n} Q_{n-1}^{\prime}(x) .
$$


The polynomials $S_{n}(x), n \geq 0$, generated by

$$
\begin{aligned}
& x S_{n}(x)=B_{n} S_{n+1}(x)+A_{n} S_{n-1}(x), \quad n \geq 0, \\
& S_{-1}(x)=0, \quad S_{0}(x)=1,
\end{aligned}
$$

are called the dual polynomials of $\left\{R_{n}(x)\right\}$. It follows from (5.14) and (5.15) that the $R_{n}(x)$ 's are random walk polynomials if and only if the relationship

$$
\frac{2^{n k-n} P_{n}^{(1)}(x)}{B_{0} \cdots B_{n-1}}=Q_{n}^{\prime}(x)=S_{n}\left(T_{k}(x)\right), \quad n \geq 0,
$$

holds. Hence,

$$
\frac{2^{n k-n} r_{n k-1}^{(1)}(x)}{B_{0} \cdots B_{n-2}}=U_{k-1}(x) S_{n-1}\left(T_{k}(x)\right), \quad n \geq 1,
$$

if and only if the $R_{n}(x)$ 's are random walk polynomials. In such a case (4.6) becomes

$$
X(x)=U_{k-1}(x) \lim _{n \rightarrow \infty} \frac{S_{n-1}\left(T_{k}(x)\right)}{R_{n}\left(T_{k}(x)\right)-R_{n-2}\left(T_{k}(x)\right)} \frac{B_{0} \cdots B_{n-2}}{A_{0} \cdots A_{n-1}},
$$

which is (5.3) of [8].

The sieved polynomials of the second kind $\left\{s_{n}(x)\right\}$ of $\left\{R_{n}(x)\right\}$ are defined through (1.14). They are the associated polynomials of order 1 of the system $\left\{q_{n}(x)\right\}$ determined by

$$
\begin{aligned}
x q_{n k+j}(x)=q_{n k+j+1}(x)+b_{n}^{(j)} q_{n k-1+j}(x) & \\
n & \geq 0, q_{-1}(x)=0, q_{0}(x)=1,
\end{aligned}
$$

where $b_{n}^{(j)}=\frac{1}{4}, n \geq 0, j=2,3, \ldots, k-1, k>2$, and

$$
b_{0}^{(0)}=b_{0}^{(1)}=\frac{1}{2}, \quad b_{n}^{(0)}=a_{n-1}^{(k-1)}=\frac{1}{2} B_{n-1}, \quad b_{n}^{(1)}=a_{n}^{(0)}=\frac{1}{2} A_{n-1}, \quad n \geq 1 .
$$

Therefore, if $\left\{P_{n}(x)\right\}$ is the system of link polynomials of $\left\{q_{n}(x)\right\}$, then

$$
s_{n k-1}(x)=2^{1-k} U_{k-1}(x) P_{n-1}^{(1)}(x) .
$$

The polynomials $P_{n}^{(1)}(x)$ satisfy $P_{-1}^{(1)}(x)=0, P_{0}^{(1)}(x)=1$, and

$$
\begin{aligned}
2^{1-k} & {\left[T_{k}(x)-\left(A_{n}+B_{n}-1\right) U_{k-2}(x)\right] P_{n}^{(1)}(x) } \\
& =P_{n+1}^{(1)}(x)+4^{1-k} A_{n-1} B_{n} P_{n-1}^{(1)}(x), \quad n \geq 0 .
\end{aligned}
$$

Hence,

$$
\frac{2^{n k-n} P_{n}^{(1)}(x)}{A_{0} \cdots A_{n-1}}=R_{n}\left(T_{k}(x)\right)
$$

that is,

$$
\frac{2^{n k-n} S_{n k-1}(x)}{A_{0} \cdots A_{n-2}}=U_{k-1}(x) R_{n-1}\left(T_{k}(x)\right), \quad n>1,
$$

if and only if the $R_{n}(x)$ 's are random walk polynomials. In such case we have

$$
\frac{2^{n k-n} P_{n}^{(2)}(x)}{A_{1} \cdots A_{n}}=R_{n}^{(1)}\left(T_{k}(x)\right),
$$


so that

$$
\frac{P_{n-1}^{(2)}(x)}{P_{n}^{(1)}(x)}=\frac{2^{1-k}}{A_{0}} \cdot \frac{R_{n-1}^{(1)}\left(T_{k}(x)\right)}{R_{n}\left(T_{k}(x)\right)},
$$

and the Stieltjes transform of the orthogonality measure of $\left\{s_{n}(x)\right\}$ is given by

$$
X(x)=\frac{2}{U_{k-1}(x)}\left\{U_{k-2}(x)+\frac{B_{0}}{A_{0}} \lim _{n \rightarrow \infty} \frac{R_{n-1}^{(1)}\left(T_{k}(x)\right)}{R_{n}\left(T_{k}(x)\right)}\right\},
$$

as follows from (4.12). Formula (5.28) was not included in [8] or [15].

It is interesting to note that (5.25) generalizes in the random walk case, and obviously in such a case only to the following relationship:

$$
\begin{array}{r}
\frac{2^{n k-k+j} s_{n k+j}(x)}{A_{0} \cdots A_{n-1}}=U_{j}(x) R_{n}\left(T_{k}(x)\right)+U_{k-j-2}(x) R_{n-1}\left(T_{k}(x)\right), \\
0<j<k .
\end{array}
$$

This follows from (2.25) and (5.25), and it is (2.3) of [8].

It is important to observe that not every system $\left\{p_{n}(x)\right\}$ of symmetric sieved polynomials of the first kind is the system of sieved polynomials of the first kind resulting from sieving a symmetric set of orthogonal polynomials. This is due to the fact that for general systems of symmetric sieved polynomials of the first kind $a_{0}^{(0)}$ and $a_{0}^{(1)}$ are arbitrary. Obviously, the arbitrariness of $a_{0}^{(0)}$ is unimportant. But $a_{0}^{(1)}$ can be taken to be $\frac{1}{2} a_{0}, a_{0}>0, a_{0} \neq 1$. Let, however, $\left\{R_{n}(x)\right\}$ be the system of orthogonal polynomials generated by

$$
\begin{aligned}
& x R_{n}(x)=A_{n} R_{n+1}(x)+B_{n} R_{n-1}(x), \quad n \geq 0, \\
& R_{-1}(x)=0, \quad R_{0}(x)=1,
\end{aligned}
$$

where

$$
A_{n}=2 a_{n+1}^{(0)}, \quad B_{n}=2 a_{n+1}^{(1)}, \quad n \geq 0 .
$$

Also, let $\left\{r_{n}(x)\right\}$ be the system of sieved polynomials of the first kind of $\left\{R_{n}(x)\right\}$ and write $P_{n}(x)=p_{n k}(x), \bar{P}_{n}(x)=r_{n k}(x)$. A simple calculation shows that the link polynomials $P_{n}(x)$ and $\bar{P}_{n}(x)$ satisfy the relationship

$$
P_{n}(x)=\bar{P}_{n}(x)+\frac{1-a_{0}}{2^{k-1}} U_{k-2}(x) \bar{P}_{n-1}^{(1)}(x)+\frac{1}{2}\left(1-a_{0}\right) \bar{P}_{n-2}^{(2)}(x) .
$$

We note that (5.32) gives $P_{n}(x)$ in terms of $\bar{P}_{n}(x), \bar{P}_{n}^{(1)}(x)$, and $\bar{P}_{n-2}^{(2)}(x)$. In practice, however, it may be more convenient to obtain $P_{n}(x)$ directly from the recurrence relation (3.5).

The system of sieved Pollaczek polynomials of the first kind, as given by (1.11), is not a system of sieved symmetric polynomials, and $a_{n}^{(0)}+a_{n}^{(1)} \neq \frac{1}{2}$ for all $n$, as long as $a \neq 0$. Their link polynomials $\left\{P_{n}(x)\right\}$ are not given by a polynomial mapping. We will show, however, that the Stieltjes transform of their orthogonality measure can be computed using the general theory of orthogonal polynomials. It is readily seen that $\left\{P_{n}(x)\right\}$ satisfies the recurrence relation

$$
\begin{aligned}
2^{1-k} & {\left[T_{k}(x)+\frac{a}{n+\lambda+a} U_{k-2}(x)\right] P_{n}(x) } \\
& =P_{n+1}(x)+4^{-k} \frac{n}{n+\lambda+a-1} \cdot \frac{n+2 \lambda-1}{n+\lambda+a} P_{n-1}(x)
\end{aligned}
$$


and the initial conditions $P_{-1}(x)=0, P_{0}(x)=1$. If we let

$$
Q_{n}(x)=\frac{2^{n k}(\lambda+a)_{n}}{n !} P_{n}(x)
$$

then $Q_{-1}(x)=0, Q_{0}(x)=1$, and

$$
\begin{aligned}
& 2\left[(n+\lambda+a) T_{k}(x)+a U_{k-2}(x)\right] Q_{n}(x) \\
& \quad=(n+1) Q_{n+1}(x)+(n+2 \lambda-1) Q_{n-1}(x), \quad n \geq 0 .
\end{aligned}
$$

Observe that $Q_{n}(x)$ does not have a representation of the form $Q_{n}(x)=$ $R_{n}\left(T_{k}(x)\right)$. However, the Stieltjes transform of the orthogonality measure of the polynomials $\left\{p_{n}(x)\right\}$ can be obtained from

$$
X(x)=\frac{1}{2^{k-1}} U_{k-1}(x) \lim _{n \rightarrow \infty} \frac{P_{n-1}^{(1)}(x)}{P_{n}(x)}
$$

by standard techniques. To do so, assume, for simplicity, that $\lambda>0$ and $0<\lambda+a<1$. Other cases of orthogonality can be treated in a similar fashion. Clearly,

$$
\frac{P_{n-1}^{(1)}(x)}{P_{n}(x)}=2^{k}(\lambda+a) \frac{Q_{n-1}^{(1)}(x)}{Q_{n}(x)}
$$

follows from

$$
Q_{n}^{(1)}(x)=\frac{2^{n k}(\lambda+a+1)_{n}}{(n+1) !} P_{n}^{(1)}(x)
$$

Hence,

$$
X(x)=2(\lambda+a) U_{k-1}(x) \lim _{n \rightarrow \infty} \frac{Q_{n-1}^{(1)}(x)}{Q_{n}(x)} .
$$

Now, a simple calculation gives

$$
\sum_{n=0}^{\infty} Q_{n}(x) t^{n}=\left(1-\frac{t}{\alpha^{k}}\right)^{A}\left(1-\frac{1}{\beta^{k}}\right)^{B},
$$

where $\alpha=\alpha(x)$ and $\beta=\beta(x)$ are given as

$$
\alpha=\alpha(x)=x+\sqrt{x^{2}-1}, \quad \beta=\beta(x)=x-\sqrt{x^{2}-1},
$$

as in $\S 2$ of [9], and

$$
\begin{aligned}
& A=-\lambda+2 a \frac{T_{k}(x)+U_{k-2}(x)}{\alpha^{k}(x)-\beta^{k}(x)}=-\lambda+a \frac{x}{\sqrt{x^{2}-1}}, \\
& B=-\lambda-2 a \frac{T_{k}(x)+U_{k-2}(x)}{\alpha^{k}(x)-\beta^{k}(x)}=-\lambda-a \frac{x}{\sqrt{x^{2}-1}} .
\end{aligned}
$$

Since $B \rightarrow-(\lambda+a)$ as $x \rightarrow+\infty$, and $0<\lambda+a<1$, Darboux's asymptotic method (Olver [16]) readily gives

$$
Q_{n}(x) \sim\left(1-\frac{\beta^{k}}{\alpha^{k}}\right)^{A} \beta^{-n k} \frac{n^{-B-1}}{\Gamma(-B)} \quad \text { as } n \rightarrow \infty
$$


for $x$ large enough. Furthermore, one can establish the generating function

$$
\begin{aligned}
\sum_{n=0}^{\infty} Q_{n}^{(1)}(x) t^{n+1}= & \left(1-\frac{t}{\alpha^{k}}\right)^{A}\left(1-\frac{t}{\beta^{k}}\right)^{B} \\
& \cdot \beta^{k} \int_{0}^{1}\left(1-\frac{\beta^{k}}{\alpha^{k}} t u\right)^{-A-1}(1-t u)^{-B-1} d u .
\end{aligned}
$$

Applying Darboux's method to the generating function (5.44) gives

$$
Q_{n-1}^{(1)}(x) \sim \beta^{k} \frac{n^{-B-1}}{\Gamma(-B)}\left(1-\frac{\beta^{k}}{\alpha^{k}}\right)^{A} \beta^{-n k} \int_{0}^{1}\left(1-\frac{\beta^{k}}{\alpha^{k}} u\right)^{-A-1}(1-u)^{-B-1} d u
$$

as $n \rightarrow \infty$, and for $x$ fixed. The asymptotic formulas (5.43) and (5.45) and analytic continuation establish the limiting relation

$$
\lim _{n \rightarrow \infty} \frac{Q_{n-1}^{(1)}(x)}{Q_{n}(x)}=\beta^{k} \int_{0}^{11}\left(1-\frac{\beta^{k}}{\alpha^{k}} u\right)^{-A-1}(1-u)^{-B-1} d u \quad \text { as } n \rightarrow \infty,
$$

which holds for $x \notin[-M, M]$, where

$$
M=\operatorname{Sup}_{n}\left\{\frac{1}{2} \sqrt{\frac{n}{\lambda+\alpha+n}}, \frac{1}{2} \sqrt{\frac{n+2 \lambda}{n+\lambda+\alpha}}\right\} .
$$

The integral in (5.46) is in the sense of the Hadamard principal value, i.e.,

$$
\int_{0}^{17}\left(1-\frac{\beta^{k}}{\alpha^{k}} u\right)^{-A-1}(1-u)^{-B-1} d u=-\frac{1}{B}{ }_{2} F_{1}\left(\begin{array}{l}
A+1,1 \mid \frac{\beta^{k}}{\alpha^{k}} \\
-B+1
\end{array}\right) .
$$

The integral in (5.46) is an analytic function of $x$ for $x \notin[-1,1]$, except at the points $x_{n}$ of $\mathbf{R}$ where $B\left(x_{n}\right)=n, n=0,1,2 \ldots$, which are simple poles. Hence,

$$
\begin{aligned}
X(x) & =2(\lambda+a) U_{k-1}(x) \beta^{k} \int_{0}^{1\rceil}\left(1-\frac{\beta^{k}}{\alpha^{k}} u\right)^{-A-1}(1-u)^{-B-1} d u \\
& =2 \frac{\lambda+a}{\alpha-\beta}\left(1-\frac{\beta^{k}}{\alpha^{k}}\right) \int_{0}^{1\rceil}\left(1-\frac{\beta^{k}}{\alpha^{k}} u\right)^{-A-1}(1-u)^{-B-1} d u,
\end{aligned}
$$

which coincides with (3.56) of [9] in the case $b=0$. As for the symmetric sieved polynomials of the second kind, they are by definition associated polynomials of order 1 of a system of sieved symmetric polynomials, but their link polynomials, which are of the form $P_{n}^{(1)}(x)$, may not have a representation of the form $P_{n}^{(1)}(x)=R_{n}\left(T_{k}(x)\right)$. This is the case, for example, of the sieved Pollaczek polynomials of the second kind, given by (1.12). However, $\lim _{n \rightarrow \infty} P_{n-1}^{(2)}(x) / P_{n}^{(1)}(x)$ can be evaluated, and thereof the Stieltjes transform can be determined via (4.12). The recurrence relation for $\left\{P_{n}^{(1)}(x)\right\}$ is

$$
\begin{aligned}
\frac{1}{2^{k-1}} & {\left[T_{k}(x)+\frac{a}{n+\lambda+a+1} U_{k-2}(x)\right] P_{n}^{(1)}(x) } \\
& =P_{n+1}^{(1)}(x)+4^{1-k} \frac{n+2 \lambda+1}{n+\lambda+a+1} \cdot \frac{n}{n+\lambda+a} P_{n-1}^{(1)}(x), \quad n \geq 0 .
\end{aligned}
$$


Hence the polynomials $Q_{n}(x)$ defined by

$$
Q_{n}(x)=\frac{2^{n k}(\lambda+a+1)_{n}}{n !} P_{n}^{(1)}(x)
$$

satisfy the three term recurrence relation

$$
\begin{aligned}
& 2\left[T_{k}(x)(n+\lambda+a+1)+a U_{k-2}(x)\right] Q_{n}(x) \\
& \quad=(n+1) Q_{n+1}(x)+(n+2 \lambda+1) Q_{n-1}(x), \quad n \geq 0 .
\end{aligned}
$$

Under the assumptions, for example, that $\lambda>0$ and $a>0$, we obtain in a small interval $(1,1+\varepsilon)$ that

$$
\begin{aligned}
\lim _{n \rightarrow \infty} \frac{P_{n-1}^{(2)}(x)}{P_{n}^{(1)}(x)} & =\lim _{n \rightarrow \infty} 2(\lambda+a+1) \frac{Q_{n}^{(1)}(x)}{Q_{n}(x)} \\
& =2(\lambda+a+1) \beta^{k} \int_{0}^{1\rceil}\left(1-\frac{\beta^{k}}{\alpha^{k}} u\right)^{-A}(1-u)^{-B} d u .
\end{aligned}
$$

This and analytic continuation identify the Stieltjes transform $X(x)$ of the orthogonality measure of $\left\{p_{n}(x)\right\}$ as

$$
X(x)=2 \frac{U_{k-2}(x)}{U_{k-1}(x)}+\frac{(1+2 \lambda) \beta^{k}}{U_{k-1}(x)} \int_{0}^{17}\left(1-\frac{\beta^{k}}{\alpha^{k}} u\right)^{-A}(1-u)^{-B} d u
$$

We have used (4.12). To simplify the integral on the right-hand side of (5.53) we use the identity

$$
\begin{aligned}
(1+2 \lambda) & \int_{0}^{1\rceil}\left(1-\frac{\beta^{k}}{\alpha^{k}} u\right)^{-A}(1-u)^{-B} d u \\
= & (-A) \int_{0}^{1\rceil}\left(1-\frac{\beta^{k}}{\alpha^{k}} u\right)^{-A-1}(1-u)^{-B} d u \\
& +(-B) \int_{0}^{1\rceil}\left(1-\frac{\beta^{k}}{\alpha^{k}} u\right)^{-A}(1-u)^{-B-1} d u
\end{aligned}
$$

and integration by parts to get

$$
\begin{aligned}
& (1+2 \lambda) \int_{0}^{1\rceil}\left(1-\frac{\beta^{k}}{\alpha^{k}} u\right)^{-A}(1-u)^{-B} d u \\
& =1-A\left(1-\frac{\beta^{k}}{\alpha^{k}}\right) \int_{0}^{17}\left(1-\frac{\beta^{k}}{\alpha^{k}} u\right)^{-A-1}(1-u)^{-B} d u,
\end{aligned}
$$

and we obtain the representation

$$
X(x)=2\left[\beta+(\beta-\alpha) A \beta^{2 k} \int_{0}^{17}\left(1-\frac{\beta^{k}}{\alpha^{k}} u\right)^{-A-1}(1-u)^{-B} d u\right],
$$

which is (3.39) of [9] (with $b=0$ ). We observe that (5.48) and (5.54) were obtained in [9] and [14] by a rather painful limiting process. This illustrates the simplicity, elegance, and power of the present approach.

Formulae (4.17)-(4.20) can be used to identify the Stieltjes transforms of the measures of orthogonality of associated sieved symmetric polynomials of higher orders. For example, if $\left\{p_{n}(x)\right\}$ is the system of sieved ultraspherical 
polynomials of the first kind, the link polynomials $\left\{P_{n}^{(l)}(x)\right\}$ of $\left\{p_{n}^{((l-1) k+i)}(x)\right\}$, $l \geq 1, i=0,1, \ldots, k-1$, satisfy the recurrence relation of the monic polynomials of $c_{n}^{\lambda}(x, l)$. The latter polynomials are the associated polynomials of $c_{n}^{\lambda}(x)$ or order $l$. Hence,

$$
c_{n}^{\lambda}(x, l)=\frac{2^{n k}(l+\lambda)_{n}}{(l+1)_{n}} P_{n}^{(l)}(x)
$$

and

$$
c_{n}^{\lambda}(x, l+1)=\frac{2^{n k}(l+\lambda+1)_{n}}{(l+2)_{n+1}} P_{n}^{(l+1)}(x) .
$$

Therefore (see [7]),

$$
F^{(l)}(x)=2^{k} \frac{\lambda+l}{l} \cdot \beta^{k} \frac{\int_{0}^{1} u^{l}\left(1-\beta^{k} u / \alpha^{k}\right)^{\lambda-1}(1-u)^{\lambda-1} d u}{\int_{0}^{1} u^{l-1}\left(1-\beta^{k} u / \alpha^{k}\right)^{\lambda-1}(1-u)^{\lambda-1} d u},
$$

where $\alpha, \beta$ are given by (5.41). If we assume, for example, that $i=1,2, \ldots$, $k-1$, then

$$
\begin{aligned}
X(x)= & \frac{2}{B}\left[\alpha^{k} U_{k-i-1}(x) \int_{0}^{1} u^{l-1}\left(1-\beta^{k} u / \alpha^{k}\right)^{\lambda-1}(1-u)^{\lambda-1} d u\right. \\
& \left.\quad+U_{i-1}(x) \int_{0}^{1} u^{l}\left(1-\beta^{k} u / \alpha^{k}\right)^{\lambda-1}(1-u)^{\lambda-1} d u\right], \\
B= & \alpha^{k} U_{k-i}(x) \int_{0}^{1} u^{l-1}\left(1-\beta^{k} u / \alpha^{k}\right)^{\lambda-1}(1-u)^{\lambda-1} d u \\
& +U_{i-2}(x) \int_{0}^{1} u^{l}\left(1-\beta^{k} u / \alpha^{k}\right)^{\lambda-1}(1-u)^{\lambda-1} d u .
\end{aligned}
$$

When $i=0, X(x)$ takes the alternate form

$$
\begin{aligned}
X(x)= & \frac{2}{l-1} \frac{(\lambda+l-1)}{C} U_{k-1}(x) \beta^{k} \int_{0}^{1\rceil} u^{l-1}\left(1-\beta^{2 k}\right)^{\lambda-1}(1-u)^{\lambda-1} d u \\
C= & \int_{0}^{1\rceil} u^{l-1}\left(1-\beta^{2 k} u\right)^{\lambda-1}(1-u)^{\lambda-1} d u \\
& +U_{k-2}(x) \int_{0}^{1} u^{l-1}\left(1-\beta^{2 k} u\right)^{\lambda-1}(1-u)^{\lambda-1} d u
\end{aligned}
$$

for $l \geq 2$, and obviously, when $l=1, X(x)$ is given by

$$
X(x)=2 \lambda \beta^{k}\left(\int_{0}^{1]}\left(1-\frac{\beta^{k}}{\alpha^{k}} u\right)^{\lambda-1}(1-u)^{\lambda-1} d u\right) U_{k-1}(x) .
$$

It is interesting to note that the orthogonality measure $d \mu$ of the system $\left\{p_{n}^{(1)}(x)\right\}$ of the numerator polynomials of the sieved ultrasphericals of the first kind has, when $\lambda>\frac{1}{2}$, mass points which are interior to $\operatorname{supp}\{d \mu\}=[-1,1]$. These are called embedded mass points. More precisely, the discrete part $d \mu_{j}$ of $d \mu$ is given by

$$
d \mu_{j}(x)=\frac{2 \lambda-1}{\lambda k} \sum_{i=1}^{k-1}\left(1-x_{i}^{2}\right) \delta\left(x-x_{i}\right) d x,
$$


where

$$
x_{i}=\cos \frac{i \pi}{k}, \quad i=1,2, \ldots, k-1,
$$

are the zeros of $U_{k-1}(x)$ and $\delta\left(x-x_{i}\right)$ are the Dirac measure with unit mass at $x=x_{i}$.

In fact, from (4.18) with $l=1$ we obtain

$$
X(x)=\frac{2}{U_{k-1}(x)}\left\{U_{k-2}(x)+\beta^{k} \frac{\int_{0}^{1} u\left(1-\beta^{k} u / \alpha^{k}\right)^{\lambda-1}(1-u)^{\lambda-1} d u}{\int_{0}^{1}\left(1-\beta^{k} u / \alpha^{k}\right)^{\lambda-1}(1-u)^{\lambda-1} d u}\right\}
$$

and the assertion will follow from the following lemma.

Lemma 5.1. Let $d \mu$ be a positive measure on $\mathbf{R}$ and let

$$
X(x)=\int_{-\infty}^{+\infty} \frac{d \mu(t)}{x-t}, \quad x \notin \operatorname{supp}\{d \mu\},
$$

be its Stieltjes transform. Let $a<c<b$ and assume that both $\lim _{\varepsilon \rightarrow 0^{+}} X(x+i \varepsilon)$ and $\lim _{\varepsilon \rightarrow 0^{+}} X(x-i \varepsilon)$ exist and are finite for $x \in[a, c) \cup(c, b]$. Let $X(x)=$ $\lim _{\varepsilon \rightarrow 0^{+}} X(x+i \varepsilon)$ for $x \in[a, c) \cup(c, b]$ and further assume that

$$
\lim _{x \rightarrow c}(x-c) X(x)=\rho .
$$

Then $d \mu$ has a mass at $x=c$ whose value is $\rho$, i.e.,

$$
\mu(\{c\})=\rho .
$$

Proof. Let $0<\varepsilon, \delta<\min \{b-c, c-a\}$, and $\gamma_{\varepsilon, \delta}$ be a positively oriented rectangular contour with center $c$ and vertices $(c \pm \delta, \pm \varepsilon)$. Integrating $X(x)$ around $\gamma_{\varepsilon, \delta}$ gives

$$
\begin{aligned}
& \int_{c-\delta}^{c+\delta}\{X(x-i \varepsilon)-X(x+i \varepsilon)\} d x \\
& \quad+i \int_{-\varepsilon}^{\varepsilon}\{X(c+\delta+i t)-X(c-\delta-i t)\} d t=\int_{\gamma_{\varepsilon, \delta}} X(x) d x .
\end{aligned}
$$

Now, the integral on the right-hand side can be replaced by $\int_{\gamma_{\delta}} X(x) d x$, where $\gamma_{\delta}$ denotes a positively oriented circle of radius $\delta$ and center at $x=c$, and the second integral on the left goes to 0 when $\varepsilon \rightarrow 0+$. If

$$
\sigma(\lambda)=\int_{-\infty}^{\lambda} d \mu, \quad \lambda \in \mathbf{R},
$$

then

$$
\begin{aligned}
\sigma(c+\delta)-\sigma(c-\delta-0) & =\lim _{\varepsilon \rightarrow 0} \frac{1}{2 \pi i} \int_{c-\delta}^{c+\delta}\{X(x-i \varepsilon)-X(x+i \varepsilon)\} d x \\
& =\frac{1}{2 \pi i} \int_{\gamma_{\delta}} X(x) d x
\end{aligned}
$$

so that

$$
\mu(\{c\})=\lim _{\delta \rightarrow 0^{+}}\{\sigma(c+\delta)-\sigma(c-\delta-0)\}=\lim _{\delta \rightarrow 0} \frac{1}{2 \pi i} \int_{\gamma_{\delta}} X(x) d x=\rho,
$$

which proves our lemma. 
Relation (5.61) now follows from the fact that when $\lambda>\frac{1}{2}$, the term in braces in (5.63) tends to $\pm(2 \lambda-1) / 2 \lambda$ when $x \rightarrow x_{k}$.

When $\lambda=1, d \mu$ takes the simple form

$$
d \mu(x)=\frac{1}{\pi} \eta(x) \sqrt{1-x^{2}} d x+\frac{1}{2} \sum_{i=1}^{k-1}\left(1-x_{i}^{2}\right) \delta\left(x-x_{i}\right) d x,
$$

where $\eta(x)$ is the characteristic function of the interval $[-1,1]$. When $k=2$, $d \mu(x)$ takes the form

$$
d \mu(x)=\frac{1}{\pi} \eta(x) \sqrt{1-x^{2}} d x+\frac{1}{2} \delta(x) d x,
$$

which shows that half of the total mass of $d \mu$ is centered at $x=0$.

Another example of a positive measure with embedded masses is in [13].

\section{CONNECTION WITH POLYNOMIAL MAPPINGS}

Let $k$ be a given positive integer and let $c \geq 2^{k-1}$. In [13], Geronimo and Van Assche proved that if $\left\{P_{n}(x)\right\}$ is a system of symmetric orthogonal polynomials, then the system of monic orthogonal polynomials $\left\{p_{n}(x)\right\}$ obtained from $\left\{P_{n}(x)\right\}$ by means of a polynomial mapping $T(x)=2^{1-k} c T_{k}(x)$, with $W(x)=2^{1-k} k^{-1} c U_{K-1}(x)$, is a system of symmetric sieved polynomials. More can be said. In fact,

Theorem 6.1. The system $\left\{p_{n}(x)\right\}$ is the set of sieved polynomials of the first kind of a set $\left\{R_{n}(x)\right\}$ of random walk polynomials.

Proof. Assume $P_{-1}(x)=0, P_{0}(x)=1$, and

$$
x P_{n}(x)=A_{n} P_{n+1}(x)+B_{n} P_{n-1}(x), \quad n \geq 0 .
$$

Then, for some constant $C_{n}>0, p_{n k}(x)=C_{n} P_{n}(T(x))$, so that

$$
\widehat{T}_{k}(x) p_{n k}(x)=\frac{C_{n} A_{n}}{C_{n+1} c} p_{n k+k}(x)+\frac{C_{n} B_{n}}{C_{n-1} c} p_{n k-k}(x), \quad n \geq 0 .
$$

Hence,

$$
\frac{C_{n+1}}{C_{n}}=\frac{A_{n}}{c}, \quad n \geq 0
$$

so that

$$
\widehat{T}_{k}(x) p_{n k}(x)=p_{n k+k}(x)+\frac{A_{n-1} B_{n}}{c^{2}} p_{n k-k}(x), \quad n \geq 0 .
$$

This implies that

$$
p_{n k}(x)=\frac{A_{0} \cdots A_{n-1}}{c^{n}} P_{n}(T(x))
$$

and if $a_{n}^{(j)}, n \geq 0, j=0,1, \ldots, k-1$, with $a_{n}^{(j)}=\frac{1}{4}, j=2, \ldots, k-1$, are the coefficients in the recurrence relation of $\left\{p_{n}(x)\right\}$, then

$$
T_{k}(x)+\left(1-2 a_{0}^{(1)}\right) U_{k-2}(x)=T_{k}(x) .
$$


This shows that

$$
a_{0}^{(1)}=\frac{1}{2} \text {. }
$$

Hence, $\left\{p_{n k}(x)\right\}$ is the system of sieved polynomials of the first kind of a symmetric system $\left\{R_{n}(x)\right\}$. Furthermore $\left\{R_{n}(x)\right\}$ is generated recursively from $R_{-1}(x)=0, R_{0}(x)=1$, and

$$
x R_{n}(x)=A_{n}^{\prime} R_{n+1}(x)+B_{n}^{\prime} R_{n-1}(x), \quad n \geq 0,
$$

where

$$
A_{n}^{\prime}=\frac{1}{2} a_{n+1}^{(0)}, \quad B_{n}^{\prime}=\frac{1}{2} a_{n+1}^{(1)}, \quad n \geq 0,
$$

as was proved in $\S 5$. Since from (6.4) it also follows that

$$
T_{k}(x)+\left(1-2 a_{n}^{(0)}-2 a_{n}^{(1)}\right) U_{k-2}(x)=T_{k}(x),
$$

then

$$
2 a_{n+1}^{(0)}+2 a_{n+1}^{(1)}=A_{n}^{\prime}+B_{n}^{\prime}=1, \quad n \geq 0,
$$

and the theorem is proved.

We observe that the $p_{n k}(x)$ 's have, in addition to (6.5), the representation

$$
p_{n k}(x)=2^{n-n k} A_{0}^{\prime} \cdots A_{n-1}^{\prime}\left\{R_{n}\left(T_{k}(x)\right)-R_{n-2}\left(T_{k}(x)\right)\right\} .
$$

It follows that a system of symmetric polynomials is the set of monic polynomials obtained from a symmetric orthogonal system by a polynomial mapping $c \widehat{T}_{k}(x), c \geq 2^{k-1}$, with $W(x)=c k^{-1} \widehat{U}_{k-1}(x)$, if and only if it is a set of sieved random walk polynomials of the first kind.

In case $k>2$, more can be said.

Theorem 6.2. If $k>2$ and $\left\{p_{n}(x)\right\}$ is a system of sieved symmetric polynomials, and if $\left\{p_{n}(x)\right\}$ can be obtained as the monic polynomials of an orthogonal set $\left\{P_{n}(x)\right\}$ by an arbitrary change of variables with polynomial mapping $T(x)$, then $\left\{p_{n}(x)\right\}$ is the system of sieved polynomials of the first kind of a set $\left\{R_{n}(x)\right\}$ of random walk polynomials, and $P_{n}(x)=Q_{n}(x+b)$, where $\left\{Q_{n}(x)\right\}$ is a system of symmetric polynomials and $b$ is a real number.

Proof. Let $\left\{p_{n}(x)\right\}$ be the system of monic polynomials obtained from $\left\{P_{n}(x)\right\}$, where $P_{-1}(x)=0, P_{0}(x)=1$, and

$$
\left(x-b_{n}\right) P_{n}(x)=A_{n} P_{n+1}(x)+B_{n} P_{n-1}(x), \quad n \geq 0,
$$

through the polynomial mapping $T(x)$. Write $T(x)=c \widehat{T}(x)$, with $\widehat{T}(x)$ monic. As before,

$$
p_{n k}(x)=c^{-n} A_{0} \cdots A_{n-1} P_{n}(T(x))
$$

so that

$$
\left(\widehat{T}(x)-c^{-1} b_{n}\right) p_{n k}(x)=p_{n k+k}(x)+c^{-2} A_{n-1} B_{n} p_{n k-k}(x), \quad n \geq 0 .
$$

Since $a_{1}^{(0)}=\frac{1}{2}$, we have $p_{k}(x)=\widehat{T}_{k}(x)$, and find $\widehat{T}_{k}(x)=\widehat{T}(x)-c^{-1} b_{0}$. Furthermore, $\widehat{T}_{k}(x)+\frac{1}{2}\left[1-2\left(a_{n}^{(0)}+a_{n}^{(1)}\right)\right] \widehat{U}_{k-2}(x)=\widehat{T}(x)-c^{-1} b_{n}$. Hence,

$$
\frac{1}{2}\left[1-2\left(a_{n}^{(0)}+a_{n}^{(1)}\right)\right] \widehat{U}_{k-2}(x)=c^{-1}\left(b_{0}-b_{n}\right) .
$$


Since $k>2$, it follows from (6.16) that

$$
2 a_{n}^{(0)}+2 a_{n}^{(1)}=1, \quad b_{n}=b_{0}, \quad n \geq 1 .
$$

Therefore the system of polynomials $\left\{R_{n}(x)\right\}$ given by (6.8) and (6.9) is a system of random walk polynomials and $\left\{p_{n}(x)\right\}$ is the corresponding system of sieved polynomials of the first kind. Since $b_{n}=b_{0}, P_{n}(x)$ can be written as $P_{n}(x)=Q_{n}\left(x-b_{0}\right)$, where $Q_{n}(x)=P_{n}\left(x+b_{0}\right)$. Clearly $Q_{-1}(x)=0$, $Q_{0}(x)=1$, and

$$
x Q_{n}(x)=A_{n} Q_{n+1}(x)+B_{n} Q_{n-1}(x), \quad n \geq 0 .
$$

This proves the theorem.

We observe that the symmetric sieved Pollaczek polynomials of the first kind cannot be obtained from a symmetric system via a polynomial mapping of the form $c 2^{1-k} T_{k}(x)$.

We finally observe that if $k=2$, the system of polynomials given by (1.1) with

$$
\begin{aligned}
& a_{0}^{(0)}=a_{0}^{(1)}=\frac{1}{2} ; \\
& a_{n}^{(0)}=\frac{n}{4(\lambda+n+a)}, \quad a_{n}^{(1)}=\frac{n+2 \lambda}{4(\lambda+n+a)}, \quad n \geq 1,
\end{aligned}
$$

is the system of monic polynomials of $\left\{P_{n}\left(T_{k}(x)\right)\right\}$, where

$$
P_{n}(x)=P_{n}^{\lambda}(x ; a, a)-2 a P_{n-1}^{\lambda}(x ; a, a ; 1)-a P_{n-2}^{\lambda}(x ; a, a ; 2), \quad n \geq 0 .
$$

In (6.20), $\left\{P_{n}^{\lambda}(x ; a, a)\right\}$ is the system of Pollaczek polynomials (see [9]), and $\left\{P_{n}^{\lambda}(x ; a, a ; i)\right\}$ is the system of the corresponding associated polynomials of order $i$. If $a \neq 0,\left\{P_{n}(x)\right\}$ is not a system of sieved random walk polynomials, and $P_{n}(x)$ cannot be written in the form $Q_{n}(x-b)$ with $Q_{n}(x)$ symmetric.

\section{ACKNOWLEDGMENTS}

The authors thank Guillermo Rodriguez of the Universidad Javeriana, Bogota, for his help. The first author thanks the Department of Mathematics at the University of South Florida for hospitality during his visit in the Spring of 1989.

\section{REFERENCES}

1. W. Al-Salam, W. R. Allaway, and R. Askey, Sieved ultraspherical polynomials, Trans. Amer. Math. Soc. 234 (1984), 39-55.

2. R. Aksey and M. E. H. Ismail, The Rogers q-ultraspherical polynomials, Approximation Theory III, Academic Press, 1980, pp. 223-278.

3. _ـ A generalization of ultraspherical polynomials, Studies in Pure Mathematics (P. Erdös, ed.), Birkhäuser, Basel, 1983, pp. 55-78.

4. - Recurrence relations, continued fractions and orthogonal polynomials, Mem. Amer. Math. Soc. No. 300 (1984).

5. R. Askey and D. P. Shukla, Sieved Jacobi polynomials (to appear).

6. R. Askey and J. Wilson, Some basic hypergeometric polynomials that generalize Jacobi polynomials, Mem. Amer. Math. Soc. No. 319 (1985).

7. J. Bustoz and M. E. H. Ismail, The associated ultraspherical polynomials and their $q$ analogues, Canad. J. Math. 33 (1982), 718-736. 
8. J. A. Charris and M. E. H. Ismail, On sieved orthogonal polynomials. II: Random walk polynomiais, Canad. J. Math. 38 (1986), 397-415.

9. __ On sieved orthogonal polynomials. V: Sieved Pollaczek polynomials, SIAM J. Math. Anal. 18 (1987), i 177-1218.

10. J. A. Charris and G. Rodriguez, On the spectral properties of a class of orthogonal polynomials, Rev. Colombiana Mat. 24 (1990), 153-177.

11. T. S. Chihara, An introduction to orthogonal polynomials, Gordon and Breach, New York, 1978.

12. J. Geronimo and W. Van Assche, Orthogonal polynomials with asymptotically periodic recurrence coefficients, J. Approx. Theory 46 (1986), 251-283.

13. __ Orthogonal polynomials on several intervals via a polynomial mapping, Trans. Amer. Math. Soc. 308 (1988), 559-581.

14. M. E. H. Ismail, On sieved orthogcnal polynomials. I: Symmetric Pollaczek analogues, SIAM J. Math Anal. 16 (1985), 89-111.

15. __ On sieved orthogonal polynomials. III: Orthogonality on several intervals, Trans. Amer. Math. Soc. 294 (1986), 89-111.

16. F. W. J. Olver, Asymptotics and special functions, Academic Press, New York, 1974.

17. L. J. Rogers, On the expansion of some infinite products, Proc. London Math. Soc. (3) 24 (1892), 337-352.

18. Second memoir on the expansion of some infinite products, Proc. London Math. Soc. (3) 25 (1894), 318-342.

19. __ Third memoir on the expansion of some infinite products, Proc. London Math. Soc. (3) 26 (1895), 15-32.

20. H. A. Slim, On co-recursive orthogonal polynomials and their application to potential scattering, J. Math. Anal. Appl. 136 (1988), 1-19.

21. J. C. Wheeler, Modified moments and continued fraction coefficients for the diatomic linear chain, J. Chem. Phys. 80 (1984), 472-476.

Department of Mathematics, University of South Florida, Tampa, Florida 33620

Current address, J. A. Charris: Department of Mathematics, The National University of Colombia, Bogotá, Colombia 